\title{
Adherence to the Nordic Nutrition Recommendations as a measure of a healthy diet and upper respiratory tract infection
}

\author{
Elinor Fondell ${ }^{1,2, *}$, Sara E Christensen ${ }^{1}$, Olle Bälter ${ }^{1,3}$ and Katarina Bälter ${ }^{1}$ \\ 'Department of Medical Epidemiology and Biostatistics, Karolinska Institutet, Nobels vag 12a, SE-171 77 \\ Stockholm, Sweden: ${ }^{2}$ Osher Center for Integrative Medicine, Karolinska Institutet, Stockholm, Sweden: \\ ${ }^{3}$ School of Computer Science and Communication, Royal Institute of Technology, Stockholm, Sweden
}

Submitted 20 March 2010: Accepted 11 August 2010: First published online 21 September 2010

\begin{abstract}
Objective: The Nordic countries have published joint dietary recommendations, the Nordic Nutrition Recommendations (NNR), since 1980. We evaluated adherence to the NNR as a measure of a healthy diet and its potential association with self-reported upper respiratory tract infection (URTI).

Design: A prospective, population-based study with a follow-up period of 4 months. Dietary intake was assessed using a semi-quantitative FFQ with ninetysix items, along with other lifestyle factors, at baseline. URTI was assessed every three weeks. A Poisson regression model was used to control for age, sex and other confounding factors.

Setting: A middle-sized county in northern Sweden.

Subjects: Swedish men and women ( $n$ 1509) aged 20-60 years.

Results: The NNR include recommendations on macronutrient proportions, physical activity and intake of micronutrients, sodium, fibre and alcohol. We found that overall adherence to the NNR was moderately good. In addition, we found that high adherence to the NNR $(>5.5$ adherence points) was not associated with a lower risk of URTI (incidence rate ratio (IRR) $0 \cdot 89$, 95\% CI $0 \cdot 73,1 \cdot 08$ ) compared with low adherence $(<4.5$ adherence points). When investigating individual components of the NNR, only high physical activity was associated with lower URTI risk (IRR $=0 \cdot 82$, $95 \%$ CI $0 \cdot 69,0 \cdot 97$ ) whereas none of the dietary components were associated with risk of URTI.

Conclusions: Overall adherence to the NNR was moderately good. Overall adherence to the NNR was not associated with URTI risk in our study. However, when investigating individual components of the NNR, we found that high physical activity was associated with lower URTI risk.
\end{abstract}

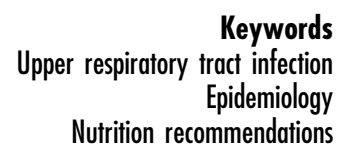

The first nutrition recommendations date back to Britain in 1862. Following economic depression, the British Privy Council wanted guidelines for food that was cheap but would avoid diseases related to malnutrition ${ }^{(1)}$. Today, most industrialized countries have nutrition recommendations and the Nordic countries have published joint recommendations, the Nordic Nutrition Recommendations $(\mathrm{NNR})^{(2)}$, since 1980 . The NNR focus on the energy distribution from macronutrients, intake of micronutrients, and the most recent version from 2004 includes physical activity. The NNR are based on the current nutritional situation in the Nordic countries and available scientific knowledge to promote overall good health and reduce the risk of diet-associated diseases.

Upper respiratory tract infection (URTI), including the common cold and influenza, is a frequent disease which, in addition to causing individual suffering, is responsible for huge costs to society. URTI is estimated to cost $\$$ US 40 billion per year in the USA ${ }^{(3)}$, not including the cost of influenza, and is the most common reason for seeking primary care in many countries ${ }^{(4)}$. Despite this, little is known about how to decrease susceptibility.

A large number of studies have focused on the effects of dietary patterns on chronic diseases, such as CVD and cancer $^{(5-9)}$. For example, a lower risk of CVD was found among women adhering to the Dietary Guidelines for Americans $^{(9)}$. Less is known about the effects of dietary patterns on respiratory infections. Most nutrient deficiencies are related to suppression of both innate and adaptive immune functions. For example, the fat-soluble vitamins $\mathrm{A}, \mathrm{D}$ and $\mathrm{E}$ have been shown to affect immune cell lineage development, as well as several immune functions including apoptosis and phagocytosis ${ }^{(10)}$. In addition, vitamin $\mathrm{E}$ supplementation in the elderly has been shown to reduce susceptibility to infection ${ }^{(11)}$, and vitamin C has since 1937 been suggested to reduce the 
risk of URTI ${ }^{(12)}$. Other nutrients suggested to affect the risk of URTI are $n$-3 fatty acids, selenium and zinc ${ }^{(10,13,14)}$. However, less is known about macronutrient composition and risk of URTI.

We aim to evaluate adherence to the NNR in a population-based, prospective web cohort and to investigate if high adherence to the NNR is associated with self-reported incidence of URTI, compared with low adherence.

Because URTI typically has a brief duration, frequent follow-ups are needed for an accurate incidence tally. We assessed self-reported incidence of URTI every third week for 15 weeks by sending an email linked to a short web questionnaire to all participants.

\section{Subjects and methods}

The LIME (Lifestyle and Immune function) study comprises 1509 men and women, aged 20-60 years, residing in a middle-sized county in northern Sweden. The study is a population-based cohort on lifestyle factors and immune function, using the Internet as a tool for data collection. Subjects were randomly selected from the Swedish Total Population Registry at Statistics Sweden, and invited to participate in the study in January 2004. The study was approved by the Research Ethics Committee at Karolinska Institutet.

\section{Study design}

Invitations to participate in the study were sent out via regular paper mail. The invitations included information on how to access the web questionnaire, details on use of a web browser, the URL to the web questionnaire, and an individual username. The baseline questionnaire about lifestyle factors included a question about the participant's email address. We sent five follow-up questionnaires during the following 15 weeks (in February, March, early April, late April and May). Every questionnaire included questions on URTI during the three preceding weeks. We sent reminders to non-responders by email 1.5 weeks after each follow-up.

Five thousand individuals were invited to participate in this prospective study, of whom 1805 completed the baseline web questionnaire in the three weeks that the web questionnaire was open. No further reminder for the baseline questionnaire was given since we aimed for a high continuation rate rather than a high initial response rate. After exclusions, 1509 were eligible for follow-up questionnaires. We excluded participants who had URTI at baseline ( $n$ 236), lacked an email address ( $n$ 17) or chose not to disclose it ( $n 20$ ). During the study it became evident to us that participants at one specific workplace had an email server that filtered our emails as junk mail. We excluded these participants from the study ( $n$ 23) since they could not be invited via email to fill out the follow-up questionnaires. Response rate for each follow-up questionnaire ranged between 83 and $84 \%$ (number of responders to that follow-up questionnaire divided by the number eligible for that follow-up questionnaire). In total, 1111 out of 1509 responded ( $74 \%$ of baseline respondents) to all five follow-ups.

The baseline web questionnaire has been described in detail elsewhere ${ }^{(15)}$. In brief, the questionnaire included immediate checks for incomplete or implausible answers, reminder messages to the respondent when a question was left unanswered, hiding of non-relevant follow-up questions, automatic summarization of answers, voluntary personalized feedback to the respondents on BMI, energy expenditure and intakes of vitamin C, calcium, fibre and iron, and illustrations to clarify complex questions. No software installation was required to complete the web questionnaire.

\section{Assessment of exposure}

Diet was assessed at baseline by a ninety-six-item validated semi-quantitative $\mathrm{FFQ}^{(16,17)}$, including questions on vitamin and mineral supplements, measuring the usual dietary intake. Consumption data from the questionnaire, including portion size, were linked to the Swedish National Food Administration database version 19/05/2009 (Uppsala, Sweden) on energy and nutrient content in various food products in order to calculate the daily mean intake. Comparing the FFQ with a $7 \mathrm{~d}$ food diary ${ }^{(16)}$, Spearman correlation coefficients ranged from 0.38 (iron) to 0.81 (vitamin $\mathrm{C}$ ) for micronutrients and had the following values for macronutrients: 0.44 (protein), 0.73 (carbohydrates), 0.71 (fibre), 0.70 (total fat), 0.75 (SFA), $0 \cdot 66$ (MUFA), $0 \cdot 49$ (PUFA) and 0.81 (alcohol). Total physical activity was also assessed at baseline. Participants were asked to estimate the amount of time (hours and minutes) spent on each out of nine activity levels on a usual day and night. Each activity level was explained by examples of activities ${ }^{(18)}$.

\section{Description of the Nordic Nutrition Recommendations}

For several decades, the Nordic countries have worked together to set dietary guidelines. The NNR are based on research data from epidemiological studies and laboratory studies, and the recommendations apply to all the Nordic countries: Sweden, Norway, Denmark, Finland and Iceland. The NNR include recommendations on total energy intake, macronutrients as a percentage of total energy intake, intakes of fibre and salt, as well as recommended daily intakes of vitamins and minerals and recommendations on physical activity. The main goal for the NNR is to set guidelines to promote good health and to prevent major chronic diseases on a population basis $^{(2)}$.

\section{Categorization of adberence}

Table 1 lists the NNR by six groups of individual recommendations: intake from (i) macronutrients (e.g. total fat, protein and carbohydrates), (ii) micronutrients (vitamins 
Table 1 The individual recommendations in the Nordic Nutrition Recommendations (NNR) and their criteria for cut-offs and adherence scores; and mean intakes and adherence in the study population: Swedish men and women ( $n$ 1509) aged 20-60 years, LIME (Lifestyle and Immune function) study, 2004

\begin{tabular}{|c|c|c|c|c|c|}
\hline \multirow[b]{2}{*}{ Recommendation in NNR } & \multicolumn{2}{|c|}{ Cut-offs and adherence scores } & \multicolumn{3}{|c|}{ Mean intakes and adherence } \\
\hline & $\begin{array}{l}\text { Recommended } \\
\text { intake }\end{array}$ & $\begin{array}{l}\text { Total } \\
\text { score }\end{array}$ & $\begin{array}{l}\text { Mean } \\
\text { intake }\end{array}$ & SD & $\begin{array}{l}\% \text { of participants } \\
\text { within NNR intake }\end{array}$ \\
\hline \multicolumn{6}{|l|}{ Macronutrient recommendation (\% of energy) } \\
\hline Saturated fat & $\leq 10$ & & $11 \cdot 4$ & $2 \cdot 5$ & 28 \\
\hline Monounsaturated fat & $10-15$ & & $9 \cdot 4$ & $1 \cdot 8$ & 36 \\
\hline Polyunsaturated fat & $5-10$ & & $3 \cdot 8$ & $0 \cdot 8$ & 7 \\
\hline Essential fatty acids & $\geq 3$ & & $3 \cdot 7$ & $0 \cdot 8$ & 83 \\
\hline Total fat & $25-35$ & $0-1$ & $26 \cdot 8$ & $4 \cdot 4$ & 64 \\
\hline Protein & $10-20$ & & $18 \cdot 0$ & $2 \cdot 6$ & 81 \\
\hline Carbohydrates & $50-60$ & & $55 \cdot 2$ & $5 \cdot 6$ & 64 \\
\hline Sugar & $\leq 10$ & & $6 \cdot 8$ & $2 \cdot 7$ & 88 \\
\hline \multicolumn{6}{|l|}{ Micronutrient recommendation } \\
\hline \multicolumn{6}{|l|}{ Vitamin recommendations for men* } \\
\hline Retinol equivalents $(\mathrm{mg} / \mathrm{d})$ & $\geq 0.9$ & & 0.5 & 0.4 & 17 \\
\hline Thiamin $(\mathrm{mg} / \mathrm{d})$ & $\geq 1 \cdot 4$ & & $1 \cdot 4$ & 0.5 & 60 \\
\hline Riboflavin (mg/d) & $\geq 1 \cdot 7$ & & $1 \cdot 7$ & 0.7 & 60 \\
\hline $\operatorname{Niacin}(\mathrm{mg} / \mathrm{d})$ & $\geq 19$ & & $16 \cdot 0$ & $6 \cdot 2$ & 38 \\
\hline Vitamin $B_{6}(\mathrm{mg} / \mathrm{d})$ & $\geq 1 \cdot 6$ & & $2 \cdot 1$ & 0.7 & 86 \\
\hline Folate $(\mathrm{mg} / \mathrm{d})$ & $\geq 0 \cdot 3$ & & 0.3 & $0 \cdot 1$ & 27 \\
\hline Vitamin $B_{12}(\mu \mathrm{g} / \mathrm{d})$ & $\geq 2$ & & $5 \cdot 6$ & $3 \cdot 4$ & 95 \\
\hline Vitamin $C(\mathrm{mg} / \mathrm{d})$ & $\geq 75$ & & $114 \cdot 0$ & $82 \cdot 7$ & 61 \\
\hline Vitamin D $(\mu \mathrm{g} / \mathrm{d})$ & $\geq 7 \cdot 5$ & & $4 \cdot 2$ & $2 \cdot 2$ & 8 \\
\hline$\alpha$-Tocopherol (mg/d) & $\geq 10$ & $0-1$ & $6 \cdot 4$ & $2 \cdot 3$ & 13 \\
\hline \multicolumn{6}{|l|}{ Mineral recommendations for men $^{*}$} \\
\hline $\mathrm{Ca}(\mathrm{g} / \mathrm{d})$ & $\geq 0.8$ & & $1 \cdot 1$ & $0 \cdot 4$ & 70 \\
\hline$P(g / d)$ & $\geq 0.6$ & & 1.5 & 0.5 & 99 \\
\hline $\operatorname{Mg}(g / d)$ & $\geq 0.35$ & & 0.4 & $0 \cdot 1$ & 62 \\
\hline $\mathrm{K}(\mathrm{g} / \mathrm{d})$ & $\geq 3.5$ & & $3 \cdot 3$ & $1 \cdot 1$ & 45 \\
\hline $\mathrm{Fe}(\mathrm{mg} / \mathrm{d})$ & $\geq 9$ & & $12 \cdot 1$ & $4 \cdot 4$ & 45 \\
\hline $\mathrm{Zn}(\mathrm{mg} / \mathrm{d})$ & $\geq 9$ & & $10 \cdot 9$ & $3 \cdot 8$ & 81 \\
\hline $\operatorname{Se}(\mu \mathrm{g} / \mathrm{d})$ & $\geq 50$ & & $33 \cdot 3$ & $13 \cdot 8$ & 16 \\
\hline lodine (not measured) & - & & - & - & - \\
\hline $\mathrm{Cu}$ (not measured) & - & & - & - & - \\
\hline \multicolumn{6}{|l|}{ Other recommendations } \\
\hline Fibre $(g / d)$ & $>25$ & $0-1$ & $25 \cdot 1$ & $10 \cdot 9$ & 42 \\
\hline Sodium recommendation for men $(\mathrm{g} / \mathrm{d})^{*}$ & $\leq 2 \cdot 8$ & $0-1$ & $2 \cdot 7$ & 0.9 & 53 \\
\hline Alcohol recommendation ( $\%$ of energy) & $\leq 5$ & $0-1$ & $3 \cdot 4$ & $3 \cdot 1$ & 78 \\
\hline \multicolumn{6}{|l|}{ Physical activity recommendationt } \\
\hline Moderate activity $(\mathrm{min} / \mathrm{d})$ and/or & $\geq 60$ & & & & \\
\hline Vigorous activity $(\mathrm{min} / \mathrm{d})$ & $\geq 30$ & $0-1$ & & & 79 \\
\hline Total score (min-max) & & $0-6$ & & & \\
\hline
\end{tabular}

*Different cut-offs were used for women for some vitamins and minerals ${ }^{(2)}$.

+Moderate activity was defined as activities corresponding to 5 MET and vigorous as activities $>6$ MET (where MET is metabolic equivalent task). The NNR advises more than $30 \mathrm{~min}$ and preferably more than $60 \mathrm{~min}$ of moderate and/or vigorous physical activity daily in addition to inactive living.

and minerals), (iii) sodium, (iv) alcohol, (v) fibre and (vi) physical activity. For each major NNR group, every individual recommendation variable was graded on a continuous scale from 0 to 1 as follows. 1 point was given to intakes within the NNR; 0 point was given to intakes below a defined lowest value and/or above a highest value (for nutrient variables, the median of the ten lowest and/or ten highest intakes among the study population was used; for physical activity, $<30 \mathrm{~min} / \mathrm{d}$ was used as lower value); and a relative score of $0-1$ points was awarded for intakes or activity levels between the recommendation level and above the defined highest and lowest values according to the following calculation (where $Y$ is the new adherence variable and $X$ is the intake or activity level).
For lower limits, $Y$ varies from 0 to 1 :

$$
\begin{gathered}
Y=(X \text {-lowest value }) /(\text { lower NNR cut-off } \\
\text { point }- \text { lowest value })
\end{gathered}
$$

For upper limits, $Y$ varies from 1 to 0 :

$$
\begin{gathered}
Y=1-[(X-\text { higher NNR cut-off point }) /(\text { highest } \\
\text { value }- \text { higher NNR cut-off point })]
\end{gathered}
$$

Summation of the scores of individual recommendations into groups of recommendation (e.g. fat, carbohydrates, vitamins and minerals) was made by summing up the individual scores and dividing the sum by the number of individual recommendations included in the group; in that way equal weight was given to each group in the final adherence score. Sodium, alcohol, fibre and physical 
activity were kept as individual recommendations. Scores from the six recommendation groups were then summarized into a total score ranging from 0 to 6 points for each person, and divided into three groups of adherence using pre-chosen arbitrary cut-off points: $<4.5$ points for low adherence (range $0 \cdot 17-4 \cdot 49$, median $4 \cdot 10$ ), 4.5-5.5 points (median 4.99) for medium adherence and $>5.5$ points (range 5.50-5.83, median 5.60) for high adherence. The group with low adherence was considered as the reference group in the subsequent analyses.

Moreover, three additional scoring models were tested in order to evaluate the effect of scoring per se. The first alternative scoring model differs from the initial model with regard to giving more weight to the score for vitamins and minerals. The second scoring model differs from the initial model by giving more weight to the scores for vitamins, minerals and individual macronutrients. Finally, the third alternative scoring model differs from the initial model by excluding recommendations for total fat intake, monounsaturated fat intake, polyunsaturated fat intake, protein intake and carbohydrate intake and only by including recommendations that are open-ended (no closed intervals; e.g. $\leq 10 \%$ of energy from saturated fat). This way, measurement error of the FFQ would have less influence on the results.

\section{Ascertainment of upper respiratory tract infection}

Self-reported URTI was assessed at baseline and in all five follow-up questionnaires. In the follow-up questionnaires, participants were asked if they currently had an infection (cold or influenza) or if they had had a new infection during the last three weeks, or since the last questionnaire. Participants were considered to have URTI if they answered 'yes' to this question. They were instructed not to count a URTI episode twice, even if it crossed over two follow-up periods. Follow-up questions about symptoms were given to all participants who reported an infection. Seven symptoms were recorded: sore throat, cough, runny nose, headache, malaise, fever and unspecified symptoms. The influenza season of 2003-2004 in Sweden was of medium intensity. Activity peaked during the last week of December 2003 and the first week of January 2004, but declined shortly after and remained low during the rest of the season, which ended in the middle of March $^{(19)}$. Influenza vaccination history was collected at baseline and updated at every follow-up. Allergy symptoms were collected in the follow-up questionnaires in April and May (the pollen season in the part of the country where the study population lived), since symptoms of pollen allergy can mimic URTI.

\section{Data analysis metbods}

We divided the number of reported URTI by person-time at risk to get incidence rates. We estimated incidence rate ratios (IRR) with 95\% confidence intervals using Poisson regression models to assess and control for age and sex along with other confounding factors. Disease-free participants contributed three weeks of time under risk for each 3-week follow-up period. A participant with no reported URTI could therefore contribute up to five 3-week periods of time at risk, a total of 15 person-weeks. Since we did not know exactly when an episode of URTI occurred or how long it lasted during the 3 -week period, we assigned to participants who reported URTI 1.5 weeks of risk time out of the 3-week follow-up period. We considered each follow-up period to be independent of previous or later periods for the same person. The correlation in the data due to repeated measurements on the same individual was investigated by using GEE (generalizing estimating equations), but no evidence of substantial correlation was found and GEE were not used in the final analysis. Therefore, we assumed that the risk of contracting URTI was independent of one's previous history of URTI.

We assessed the confounding potential of a large number of potential confounding factors by eliminating one factor at a time from the full model. We included in the final Poisson model those that changed the effect estimate more than $10 \%$ when dropped. Age, sex, BMI, asthma, selfreported weakened immune system, month, energy intake and education all had small confounding effects, but nevertheless were kept in all multivariable models. Perceived stress and smoking status changed the estimates by more than $10 \%$ in all NNR adherence score models and were included in multivariable analyses. We also assessed as potential confounders snuff use, contact with small children, contact with large crowds at leisure time, use of public transport, poor sleep, influenza vaccination history and pollen allergy. None of these changed the estimate by more than $10 \%$ and was not included in the final model. Individuals with missing information on the NNR score or any covariate in the final multivariable model were excluded from the analyses ( $n$ 254). The multivariable analyses on the initial NNR adherence scoring model included 15780 person-weeks contributed by 1255 participants, generating 1090 cases combined ( $0-5$ cases per participant).

We fit a cubic spline model for the initial NNR adherence scoring model with six knots (at minimum and maximum, and at adherence scores of $1 \cdot 0,2 \cdot 0,3 \cdot 0$ and $4 \cdot 0)^{(20)}$ to characterize any shape of a possible dose-response relationship. We present a graph of the rates at the baseline value for all other covariates predicted from the model.

The analyses were conducted using SAS version $9 \cdot 2$ (SAS Institute, Cary, NC, USA) and Stata Intercooled version $10 \cdot 1$ (Stata Corporation, College Station, TX, USA) statistical software packages.

\section{Results}

We found only small differences in baseline characteristics between study participants regarding age, sex, BMI, asthma and energy intake. Higher adherence to the NNR 
Table 2 Baseline characteristics of study participants by adherence to the Nordic Nutrition Recommendations (NNR): Swedish men and women ( $n$ 1509) aged 20-60 years, LIME (Lifestyle and Immune function) study, 2004

\begin{tabular}{|c|c|c|c|c|c|c|}
\hline & \multicolumn{6}{|c|}{ Adherence to the NNR (initial scoring model) (score 0-6) } \\
\hline & \multicolumn{2}{|c|}{$\begin{array}{l}\text { Low adherence } \\
<4.5 \text { points }\end{array}$} & \multicolumn{2}{|c|}{$\begin{array}{l}\text { Medium adherence } \\
4 \cdot 5-5.5 \text { points }\end{array}$} & \multicolumn{2}{|c|}{$\begin{array}{l}\text { High adherence } \\
>5.5 \text { points }\end{array}$} \\
\hline \multirow[t]{2}{*}{ No. of participants } & \multicolumn{2}{|c|}{488} & \multicolumn{2}{|c|}{687} & \multicolumn{2}{|c|}{175} \\
\hline & \multicolumn{2}{|c|}{$\%$} & \multicolumn{2}{|c|}{$\%$} & \multicolumn{2}{|c|}{$\%$} \\
\hline \multicolumn{7}{|l|}{ Age (years) } \\
\hline $20-29$ & & \multicolumn{2}{|c|}{31} & \multicolumn{2}{|c|}{29} \\
\hline $30-39$ & \multirow{2}{*}{\multicolumn{2}{|c|}{$\begin{array}{l}26 \\
19\end{array}$}} & \multirow{2}{*}{\multicolumn{2}{|c|}{$\begin{array}{l}27 \\
19\end{array}$}} & \multicolumn{2}{|c|}{21} \\
\hline $40-49$ & & & & & & \\
\hline $50-60$ & \multicolumn{2}{|c|}{22} & \multicolumn{2}{|c|}{23} & \multicolumn{2}{|c|}{27} \\
\hline Sex (male) & \multirow{2}{*}{\multicolumn{2}{|c|}{41}} & \multirow{2}{*}{\multicolumn{2}{|c|}{47}} & & \\
\hline BMI & & & & & & \\
\hline Low-normal $\left(<25 \mathrm{~kg} / \mathrm{m}^{2}\right)$ & \multirow{2}{*}{\multicolumn{2}{|c|}{$\begin{array}{l}56 \\
44\end{array}$}} & \multicolumn{2}{|c|}{56} & \multicolumn{2}{|c|}{60} \\
\hline High-very high $\left(>25 \mathrm{~kg} / \mathrm{m}^{2}\right)$ & & & \multicolumn{2}{|c|}{44} & \multicolumn{2}{|c|}{40} \\
\hline \multicolumn{5}{|l|}{ Chronic stress level } & & \\
\hline Low ( $<23$, below median) & & & & & & \\
\hline High ( $>23$, above median) & & & & & & \\
\hline Education & & & & & & \\
\hline Secondary school or less & & & & & & \\
\hline University & & & & & & \\
\hline Smoking & & & & & & \\
\hline Current smoker & & & & & & \\
\hline Previous smoker & & & & & & \\
\hline Never smoker & & & & & & \\
\hline Asthma (yes) & & & & & & \\
\hline & Mean & SD & Mean & SD & Mean & SD \\
\hline Energy intake $(\mathrm{kJ} / \mathrm{d})$ & 7261 & 2582 & 7801 & 2101 & 7834 & 1559 \\
\hline Carbohydrate intake $(\mathrm{g} / \mathrm{d})$ & 224 & 84 & 244 & 66 & 251 & 50 \\
\hline Protein intake $(\mathrm{g} / \mathrm{d})$ & 75 & 33 & 81 & 26 & 80 & 17 \\
\hline Saturated fat intake $(\mathrm{g} / \mathrm{d})$ & 21 & 9 & 23 & 8 & 23 & 7 \\
\hline
\end{tabular}

appeared to be associated with lower perceived stress, lower education level and fewer smokers (Table 2).

Adherence to each dietary recommendation is shown in Table 1. PUFA and vitamin D showed the least adherence: $7 \%$ of participants were within the recommended interval for PUFA and only $8 \%$ reached the recommended intake level for vitamin D. Mean intake for PUFA was 3.8 (SD 0.8$) \%$ of total energy intake, which is well below the recommended intake of 5-10\% of energy. Observed intake for vitamin $\mathrm{D}$ was also well below the recommended intake of $7.5 \mu \mathrm{g} / \mathrm{d}$, with an observed mean of $4 \cdot 2$ (SD 2.2) $\mu \mathrm{g} / \mathrm{d}$. Participants also scored lower for $\alpha$ tocopherol (13\%) and selenium (16\%), where the mean intake was $6.4(\mathrm{SD} 2.3) \mathrm{mg} / \mathrm{d}$ and $33.3(\mathrm{SD} 13.8) \mu \mathrm{g} / \mathrm{d}$, respectively, much lower than the recommended intakes of $10 \mathrm{mg} / \mathrm{d}$ (for men) and $50 \mu \mathrm{g} / \mathrm{d}$ (for men), respectively (Table 1). A previous validation study for dietary variables of the FFQ found that Spearman correlations were moderate for PUFA $(0.49)$ and vitamin D $(0 \cdot 48)$, low for $\alpha$ tocopherol $(0 \cdot 37)$ and high for selenium $(0 \cdot 72)^{(16)}$. Almost all participants (99\%) reached the recommended intake level of phosphorus of $0.6 \mathrm{~g} / \mathrm{d}$ and almost as many (95\%) reached the recommended intake of vitamin $B_{12}$ of $2 \mu \mathrm{g} / \mathrm{d}$. For phosphorus and vitamin $\mathrm{B}_{12}$, the mean intakes $(1.5$ (sD 0.5$) \mathrm{g} / \mathrm{d}$ and 5.6 (sD 3.4$) \mu \mathrm{g} / \mathrm{d}$ ) were almost three times higher than the recommended intakes (Table 1).
We found that $50 \%$ of study participants had medium adherence ( $4 \cdot 5-5 \cdot 5$ points) and $13 \%$ had high adherence ( $>5.5$ points) to the NNR according to the initial scoring model (Table 2). Using the initial scoring model, high adherence to the NNR ( $>5.5$ points) was not associated with lower risk of URTI compared with low adherence $(<4.5$ points; IRR $=0.89,95 \%$ CI $0.73,1 \cdot 08$; Table 3$)$. In order to evaluate the effect of different scoring models for adherence, we tested three additional scoring models, see Table 3. However, the results did not change much. This may partially be explained by the correlation coefficients between the initial scoring models and the alternative scoring models being relatively high, as expected, ranging from $0 \cdot 75$ to $0 \cdot 85$.

Using the same scoring criteria as the initial model we excluded those with daily or weekly multivitamin use (about $12 \%$ of all participants; IRR $=0 \cdot 93,95 \%$ CI $0 \cdot 76$, $1 \cdot 15$ ), but the results were similar to the initial model including all multivitamin supplement users for high adherence to the NNR ( $>5.5$ points) compared with low adherence $(<4.5$ points).

Adherence to the different groups of recommendations, according to the initial scoring model, and risk of URTI is shown in Table 4. We found no association between URTI and adherence to any of the groups of recommendations. The carbohydrate group (including 
Table 3 Initial and alternative scoring models of adherence to the Nordic Nutrition Recommendations (NNR) and risk of upper respiratory tract infection among Swedish men and women ( $n$ 1509) aged 20-60 years, LIME (Lifestyle and Immune function) study, 2004

\begin{tabular}{|c|c|c|c|c|c|c|}
\hline & \multicolumn{4}{|c|}{ Adjusted for age and sex } & \multicolumn{2}{|c|}{ Multivariable model } \\
\hline & Cases & Person-weeks & IRR & $95 \% \mathrm{Cl}$ & $\mathrm{IRR}^{*}$ & $95 \% \mathrm{Cl}$ \\
\hline \multicolumn{7}{|c|}{ Adherence to NNR, initial scoring modelt (score 0-6) } \\
\hline Low ( $<4.5$ points) & 242 & 3285 & $1 \cdot 00$ & _ & $1 \cdot 00$ & _ \\
\hline Medium ( $4 \cdot 5-5 \cdot 5$ points) & 686 & 9867 & 0.91 & $0.80,1.03$ & 0.88 & $0 \cdot 77,1 \cdot 01$ \\
\hline High ( $>5.5$ points) & 206 & 3246 & 0.93 & $0 \cdot 78,1 \cdot 12$ & 0.89 & $0 \cdot 73,1.08$ \\
\hline \multicolumn{7}{|c|}{ Adherence to NNR, alternative scoring model $1 \ddagger$ (score $0-7$ ) } \\
\hline Low (<5.0 points) & 155 & 2293.5 & $1 \cdot 00$ & - & $1 \cdot 00$ & - \\
\hline Medium (5.0-6.0 points) & 518 & 7038 & $1 \cdot 02$ & $0 \cdot 88,1 \cdot 18$ & 0.98 & $0 \cdot 83,1 \cdot 15$ \\
\hline High (>6.0 points) & 461 & $7066 \cdot 5$ & 0.97 & $0 \cdot 83,1 \cdot 14$ & 0.91 & $0 \cdot 76,1 \cdot 09$ \\
\hline \multicolumn{7}{|c|}{ Adherence to NNR, alternative scoring model $2 \S$ (score $0-9$ ) } \\
\hline Low ( $<7.0$ points) & 319 & $4456 \cdot 5$ & 1.00 & - & $1 \cdot 00$ & - \\
\hline Medium ( $7 \cdot 0-8 \cdot 0$ points) & 590 & 8451 & 0.92 & $0.81,1.04$ & 0.88 & $0 \cdot 77,1 \cdot 01$ \\
\hline High ( $>8.0$ points $)$ & 225 & $3490 \cdot 5$ & 0.96 & $0 \cdot 81,1 \cdot 13$ & 0.89 & $0.74,1.08$ \\
\hline \multicolumn{7}{|c|}{$\begin{array}{l}\text { Adherence to NNR, alternative scoring model } 3 \| \text { (score 0-8) } \\
\text { (only including macronutrient recommendations } \\
\text { with open-ended cut-offs) }\end{array}$} \\
\hline Low $(<6.0$ points $)$ & 144 & 2043 & $1 \cdot 00$ & - & $1 \cdot 00$ & - \\
\hline Medium (6.0-7.0 points) & 472 & 6393 & 1.03 & $0 \cdot 89,1 \cdot 20$ & 0.98 & $0 \cdot 83,1 \cdot 16$ \\
\hline High ( $>7.0$ points) & 518 & 7962 & 0.98 & $0 \cdot 84,1 \cdot 15$ & 0.91 & $0.76,1.09$ \\
\hline
\end{tabular}

IRR, incidence rate ratio.

${ }^{*}$ IRR for all adherence scores were adjusted for age (20-29, 30-39, 40-49 and 50-60 years), sex, energy intake (in four categories), BMI (low, normal, overweight and obese), weakened immune system (yes/no), asthma (yes/no), perceived stress (below and above median), education level (secondary school or less and university), smoking (daily/less frequent/previous/never) and month (February to May).

tScoring was calculated by awarding 1 point (1p) for adherence to the following recommendation subgroups in NNR: physical activity (1p), fibre intake (1p), sodium intake (1p), alcohol intake (1p), mineral and vitamin intake (1p), and macronutrient intake (1p).

¥Scoring was calculated by awarding 1 point for adherence to each of the following recommendation subgroups in NNR: physical activity (1p), fibre intake (1p), sodium intake (1p), alcohol intake (1p), mineral (1p) and vitamin intake (1p), and macronutrient intake (1p).

§Scoring was calculated by awarding 1 point for adherence to the following recommendation subgroups in NNR: physical activity (1p), fibre intake (1p), sodium intake (1p), alcohol intake (1p), mineral (1p) and vitamin intake (1p); and 1 point to each subgroup in macronutrient recommendations: carbohydrate and sugar intake (1p), total fat intake, saturated fat intake, essential fat intake, polyunsaturated fat intake, monounsaturated fat intake (1p) and protein intake (1p).

IScoring was calculated by awarding 1 point for adherence to open-ended (no intervals) recommendation subgroups in NNR: physical activity (1p), fibre intake $(1 p)$, sodium intake (1p), alcohol intake (1p), mineral (1p) and vitamin intake (1p); and 1 point to each subgroup in macronutrient recommendations; sugar intake (1p), saturated fat intake, essential fat intake (1p) and protein intake (1p).

sugar), protein group, physical activity group and alcohol group were not included in Table 4 , since $>75 \%$ of participants fulfilled the recommendations for these groups and would therefore make comparisons difficult.

In Table 5 we show associations between URTI and absolute intake of individual recommendations, rather than adherence score, for macronutrients, sodium, fibre and physical activity. We found no association for any individual group, except for physical activity, which was associated with reduced risk of URTI (IRR $=0 \cdot 82,95 \% \mathrm{CI}$ $0 \cdot 69,0 \cdot 97)$. Single vitamins and minerals were not included in the analysis due to the large number of individual vitamins and minerals.

To study the possible continuous relationship between adherence to the NNR and URTI risk, we fit spline regressions for the initial scoring model (shown in Fig. 1). We also fit spline regressions for each group of individual NNR and URTI risk. Only physical activity appeared to be associated with URTI risk (Fig. 2).

\section{Discussion}

We found that high adherence to the NNR was not associated with risk of URTI, compared with low adherence. Different scoring models were used to assess adherence to the NNR, but the result did not differ between the scoring models. However, when analysing different parts of the NNR score, we found that high physical activity was associated with lower risk of URTI but none of the recommendations on diet were associated with risk of URTI. The study also demonstrates that the overall adherence to the NNR was moderately good.

To the best of our knowledge, there are no other studies available that have studied the effect of dietary recommendations on the risk of URTI. Previous studies evaluating dietary recommendations have focused on the effect on diseases such as myocardial infarction or cancer. McCullough et $a l^{(5,6)}$ evaluated the adherence to the Dietary Guidelines for Americans (DGA) using ten equally weighted groups (e.g. servings of grain, fruit and meat). They found no preventive effect for cancer and no effect on CVD in women $^{(6)}$ and only a small reduced risk for men ${ }^{(5)}$. A more recent study, using a continuous scoring model similar to ours, found an association between DGA and atherosclerosis progression in women ${ }^{(9)}$. It is possible that the use of food groups in recommendations such as DGA is more efficient in predicting disease than the NNR which emphasize macronutrient proportions.

In the present study, diet was assessed by a validated semi-quantitative FFQ. However, we acknowledge the limitations of FFQ. The FFQ does not assess the entire 
Table 4 Adherence score (0-1) for each group of recommendations of the Nordic Nutrition Recommendations (NNR) and risk of upper respiratory tract infection among Swedish men and women $(n$ 1509) aged 20-60 years, LIME (Lifestyle and Immune function) study, 2004

\begin{tabular}{|c|c|c|c|c|c|c|}
\hline \multirow{2}{*}{$\begin{array}{l}\text { Categorization of adherence score for each group } \\
\text { of recommendation of the NNR* }\end{array}$} & \multicolumn{4}{|c|}{ Adjusted for age and sex } & \multicolumn{2}{|c|}{ Multivariable model } \\
\hline & Cases & Person-weeks & IRR & $95 \% \mathrm{Cl}$ & IRRt & $95 \% \mathrm{Cl}$ \\
\hline \multicolumn{7}{|l|}{ Fat group $\ddagger$ intake (adherence score, $0-1$ ) } \\
\hline Low $(<0.50)$ & 267 & $3457 \cdot 5$ & $1 \cdot 00$ & - & 1.00 & - \\
\hline Medium $(0.50-0.60)$ & 487 & $6898 \cdot 5$ & 0.97 & $0 \cdot 84,1 \cdot 13$ & 0.96 & $0 \cdot 82,1 \cdot 12$ \\
\hline High $(>0.60)$ & 404 & 6354 & 0.92 & $0.78,1.08$ & 0.91 & $0.78,1.08$ \\
\hline \multicolumn{7}{|l|}{ Vitamin group intake (adherence score, $0-1$ ) } \\
\hline Low $(<0.60)$ & 414 & 5772 & $1 \cdot 00$ & - & $1 \cdot 00$ & - \\
\hline Medium $(0.60-0.90)$ & 539 & $7918 \cdot 5$ & 0.98 & $0 \cdot 86,1 \cdot 12$ & 0.93 & $0 \cdot 78,1 \cdot 10$ \\
\hline High $(>0.90)$ & 205 & $3019 \cdot 5$ & 1.05 & $0 \cdot 89,1 \cdot 25$ & 0.97 & $0 \cdot 77,1 \cdot 24$ \\
\hline \multicolumn{7}{|l|}{ Mineral group intake (adherence score, $0-1$ ) } \\
\hline Low $(<0.75)$ & 277 & $4045 \cdot 5$ & 1.00 & - & $1 \cdot 00$ & - \\
\hline Medium $(0.75-0.95)$ & 558 & 7938 & $1 \cdot 07$ & $0.93,1.24$ & 1.08 & $0.90,1.30$ \\
\hline High $(>0.95)$ & 323 & $4726 \cdot 5$ & $1 \cdot 11$ & $0.94,1.30$ & $1 \cdot 08$ & $0.85,1.37$ \\
\hline \multicolumn{7}{|l|}{ Sodium intake (adherence score, $0-1$ ) } \\
\hline $\operatorname{Low}(<0 \cdot 80)$ & 288 & 3870 & 1.00 & - & 1.00 & - \\
\hline Medium $(0 \cdot 80-<1 \cdot 00)$ & 252 & 3645 & 0.96 & $0 \cdot 81,1 \cdot 13$ & 0.97 & $0 \cdot 80,1 \cdot 18$ \\
\hline High $(1.00)$ & 618 & 9195 & 0.92 & $0.80,1.06$ & 0.93 & $0 \cdot 74,1 \cdot 17$ \\
\hline \multicolumn{7}{|l|}{ Fibre intake (adherence score, $0-1$ ) } \\
\hline Low $(<0.50)$ & 245 & $3394 \cdot 5$ & $1 \cdot 00$ & - & $1 \cdot 00$ & _ \\
\hline Medium $(0.50-1.00)$ & 450 & 6432 & $1 \cdot 00$ & $0 \cdot 86,1 \cdot 17$ & 0.97 & $0 \cdot 82,1 \cdot 14$ \\
\hline High $(1.00)$ & 463 & $6883 \cdot 5$ & $1 \cdot 03$ & $0.88,1.20$ & 0.96 & $0 \cdot 80,1 \cdot 16$ \\
\hline
\end{tabular}

IRR, incidence rate ratio.

${ }^{*}$ Carbohydrate group (including sugar), protein, physical activity and alcohol were not included in this table since $>75 \%$ of participants were within recommendations (adherence score $=1$ ) for those groups.

tIRR for all adherence scores were adjusted for age (20-29, 30-39, 40-49 and 50-60 years), sex, energy intake (in four categories), BMI (low, normal, overweight and obese), weakened immune system (yes/no), asthma (yes/no), perceived stress (below and above median), education level (secondary school or less and university), smoking (daily/less frequent/previous/never) and month (February to May).

flncluding total fat intake, saturated fat intake, essential fat intake, polyunsaturated fat intake and monounsaturated fat intake.

Table 5 Absolute cut-off points for individual recommendations of the Nordic Nutrition Recommendations (NNR) and risk of upper respiratory tract infection among Swedish men and women $(n$ 1509) aged 20-60 years, LIME (Lifestyle and Immune function) study, 2004

\begin{tabular}{|c|c|c|c|c|c|c|}
\hline \multirow[b]{2}{*}{ Intake of individual recommendations of the $\mathrm{NNR}^{*}$} & \multicolumn{4}{|c|}{ Adjusted for age and sex } & \multicolumn{2}{|c|}{ Multivariable model } \\
\hline & Cases & Person-weeks & IRR & $95 \% \mathrm{Cl}$ & IRRt & $95 \% \mathrm{Cl}$ \\
\hline \multicolumn{7}{|l|}{ Saturated fat intake (\% of energy) } \\
\hline$<10$ & 315 & $4636 \cdot 5$ & $1 \cdot 00$ & - & $1 \cdot 00$ & - \\
\hline $10-12$ & 393 & $5485 \cdot 5$ & $1 \cdot 07$ & $0.92,1 \cdot 24$ & $1 \cdot 09$ & $0.93,1.27$ \\
\hline$>12$ & 450 & 6588 & $1 \cdot 03$ & $0 \cdot 89,1 \cdot 19$ & $1 \cdot 05$ & $0 \cdot 90,1 \cdot 22$ \\
\hline \multicolumn{7}{|l|}{ Protein intake (\% of energy) } \\
\hline$<17$ & 421 & $5815 \cdot 5$ & $1 \cdot 00$ & - & $1 \cdot 00$ & - \\
\hline $17-20$ & 533 & $7615 \cdot 5$ & $1 \cdot 02$ & $0 \cdot 89,1 \cdot 16$ & $1 \cdot 02$ & $0 \cdot 89,1 \cdot 16$ \\
\hline$>20$ & 204 & 3279 & 0.94 & $0 \cdot 79,1 \cdot 12$ & 0.94 & $0 \cdot 78,1 \cdot 11$ \\
\hline \multicolumn{7}{|l|}{ Carbohydrate intake (\% of energy) } \\
\hline$<50$ & 195 & $2806 \cdot 5$ & $1 \cdot 00$ & - & $1 \cdot 00$ & - \\
\hline $50-60$ & 725 & $10780 \cdot 5$ & 0.93 & $0.79,1.09$ & 0.92 & $0.78,1.09$ \\
\hline$>60$ & 238 & 3123 & 0.98 & $0 \cdot 80,1 \cdot 19$ & 0.96 & $0 \cdot 78,1 \cdot 17$ \\
\hline \multicolumn{7}{|l|}{ Sodium intake (mg/d) } \\
\hline$<2000$ & 313 & $4258 \cdot 5$ & $1 \cdot 00$ & - & $1 \cdot 00$ & - \\
\hline 2000-3800 & 661 & $9925 \cdot 5$ & 0.96 & $0 \cdot 83,1 \cdot 10$ & $0 \cdot 87$ & $0 \cdot 71,1 \cdot 06$ \\
\hline$>3800$ & 184 & 2526 & $1 \cdot 01$ & $0.83,1.23$ & $0 \cdot 85$ & $0.63,1 \cdot 14$ \\
\hline \multicolumn{7}{|l|}{ Alcohol intake (\% of energy) } \\
\hline$<2.5$ & 586 & 8094 & $1 \cdot 00$ & - & $1 \cdot 00$ & - \\
\hline $2 \cdot 5-5 \cdot 0$ & 328 & 4896 & 0.99 & $0 \cdot 86,1 \cdot 14$ & 0.99 & $0 \cdot 86,1 \cdot 14$ \\
\hline$>5 \cdot 0$ & 244 & 3720 & $1 \cdot 02$ & $0 \cdot 88,1 \cdot 19$ & $1 \cdot 05$ & $0 \cdot 87,1 \cdot 12$ \\
\hline \multicolumn{7}{|l|}{ Fibre intake (g/d) } \\
\hline$<25$ & 695 & $9826 \cdot 5$ & $1 \cdot 00$ & - & $1 \cdot 00$ & - \\
\hline 25-35 & 302 & 4518 & $1 \cdot 02$ & $1 \cdot 17,1 \cdot 04$ & 0.99 & $0 \cdot 86,1 \cdot 15$ \\
\hline$>35$ & 161 & $2365 \cdot 5$ & $1 \cdot 04$ & $0 \cdot 88,1 \cdot 24$ & 0.98 & $0 \cdot 80,1 \cdot 19$ \\
\hline \multicolumn{7}{|l|}{ Moderateł and/or vigorous physical activity (min/d) } \\
\hline$<60$ & 522 & 7062 & $1 \cdot 00$ & - & $1 \cdot 00$ & - \\
\hline $60-120$ & 281 & $3940 \cdot 5$ & 0.90 & $0 \cdot 78,1 \cdot 05$ & 0.89 & $0.77,1 \cdot 05$ \\
\hline$>120$ & 378 & 5982 & 0.86 & $0 \cdot 74,1 \cdot 01$ & 0.82 & $0.69,0.97$ \\
\hline
\end{tabular}

IRR, incidence rate ratio.

* Single vitamins or minerals were not studied since they were too many in number and would increase the risk of chance findings.

IIRR for all adherence scores were adjusted for age (20-29, 30-39, 40-49 and 50-60 years), sex, energy intake (in four categories), BMI (low, normal, overweight and obese), weakened immune system (yes/no), asthma (yes/no), perceived stress (below and above median), education level (secondary school or less and university), smoking (daily/less frequent/previous/never) and month (February to May).

$\ddagger$ Moderate physical activity cut-offs: $<2 \mathrm{~h}, 2-3 \mathrm{~h},>3 \mathrm{~h}$ 


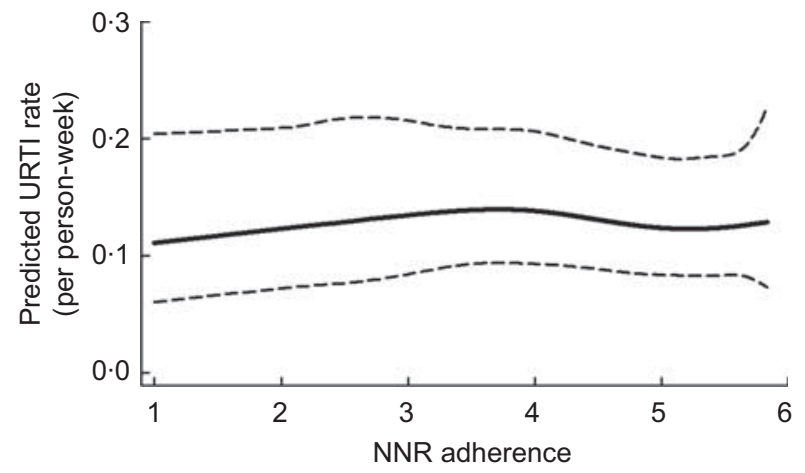

Fig. 1 Spline regressions for overall adherence to the Nordic Nutrition Recommendations (NNR) expressed as adherence score and risk of upper respiratory tract infection (URTI) among Swedish men and women ( $n$ 1509) aged 20-60 years, LIME (Lifestyle and Immune function) study, 2004. -- , $95 \%$ confidence intervals

(a)

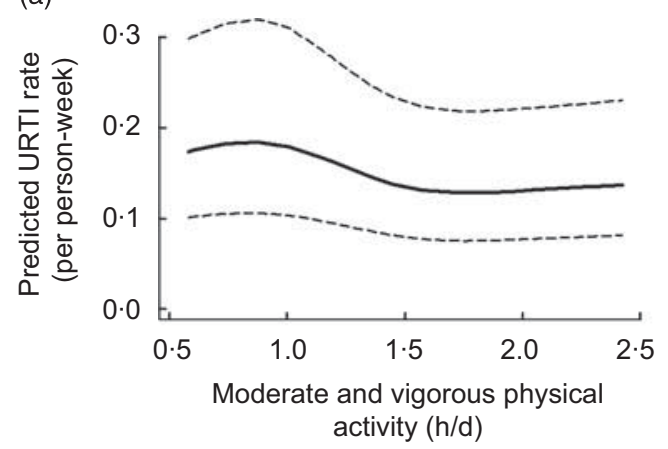

(c)

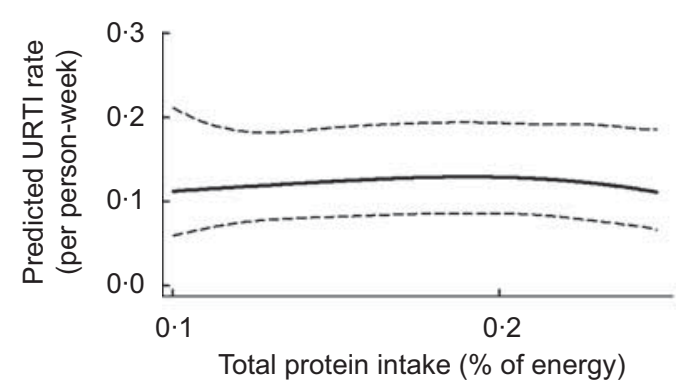

(e)

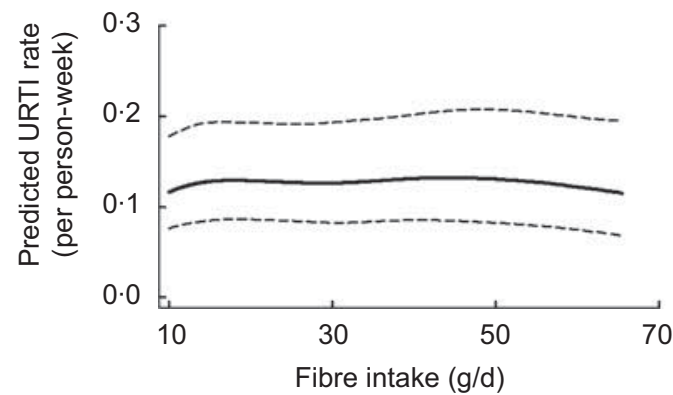

diet, but large parts of the dietary intake. The shortcomings of an FFQ can blunt a true association ${ }^{(21)}$ and thus the results from the current study should be interpreted with caution. For example, in the European Prospective Investigation into Cancer and Nutrition, which used both FFQ and food diaries, a stronger association was found between saturated fat and breast cancer on data from food diaries compared with $\mathrm{FFQ}^{(22)}$. In addition, a previous validation study using the same FFQ and repeated records of total dietary intake found that the correlation coefficients between FFQ and dietary records were moderate to high for different nutrients (ranging from 0.31 for iron to $0 \cdot 81$ for vitamin C). Still, in dietary surveys, regardless of dietary assessment method, fat (especially saturated fat) and carbohydrates are considered to be typically under-reported, while protein is typically over-reported ${ }^{(23)}$. Obese and weight-conscious

(b)

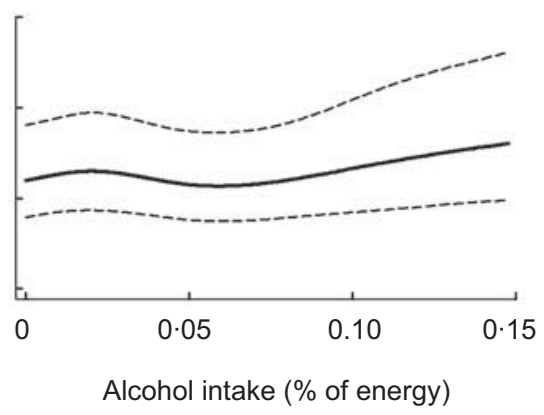

(d)

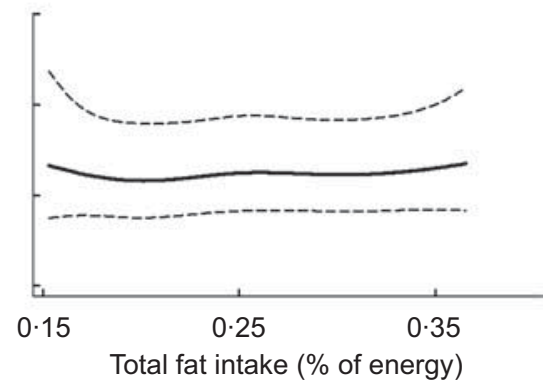

(f)

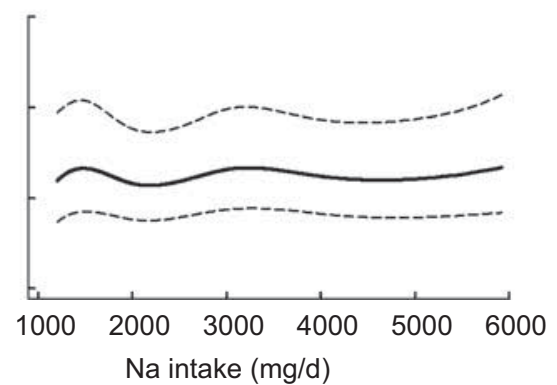

Fig. 2 Spline regressions for groups of individual recommendations in the Nordic Nutrition Recommendations (NNR) using absolute intake and risk of upper respiratory tract infection (URTI) among Swedish men and women ( $n$ 1509) aged 20-60 years, LIME (Lifestyle and Immune function) study, 2004. -.., $95 \%$ confidence intervals 
participants are considered to be those who under-report unhealthy food the most ${ }^{(24-26)}$. The intakes of various nutrients in the present study were of the same magnitude as intakes reported in a previous national Swedish study, Riksmaten, from 1997-1998, of 1215 Swedish men and women $^{(27)}$. In Riksmaten, the participants were asked to record their total intake of food and beverages using a $7 \mathrm{~d}$ food dairy and did not use an FFQ. A more precise measure of some dietary intakes would have been to use biomarkers, which were not available in our study.

Moderate validity for certain food items or nutrients may result in bias, with double-ended macronutrient recommendations (e.g. 50-60\% of energy from carbohydrates) being more vulnerable to poor validity (i.e. misclassification when using FFQ). In alternative scoring model 3 we excluded all double-ended recommendations, but the results did not change much compared with the initial scoring model. In order to reduce bias from misclassification using absolute cut-off points for the NNR scoring, we used a continuous score proportional to the distance from the guidelines (in all scoring models). By doing this, intakes close to, but not within the recommended intake were still given a high score.

We cannot rule out the possibility that the null results for overall adherence to the NNR in the present study are due to participants being generally very well-nourished, leading to a limited variation and skewing towards higher adherence scores for certain recommendations in order to see an association with URTI. Nevertheless, small variations in the scoring were taken into account by using a continuous grading scale for intakes close to NNR cut-offs. Our results on physical activity and URTI are supported by previous studies which have suggested a protective association on URTI of moderate levels of physical activity ${ }^{(28)}$, possibly mediated by a higher circulating number of natural killer cells found in resting athletes ${ }^{(29)}$. Although we cannot rule out a potential effect of reverse causality, to minimize this possibility we assessed exposure before outcome (i.e. baseline) and thereafter excluded those with URTI at baseline.

Major strengths of our study include its population-based design and the comprehensiveness of case identification using frequent follow-ups. Also, the continuation rate for each follow-up was high, i.e. more than $80 \%$ of the participants responded to each follow-up web questionnaire. Incidence of URTI was self-reported and selfdiagnosis of URTI has been shown to be reliable in adult patients ${ }^{(30)}$. The prospective nature of the study makes any potential recall bias highly unlikely. We adjusted for several potential confounders in the model, including stress, smoking, education level and season, observing no great difference between the age-adjusted and the multivariable models, but unmeasured aspects of a healthy diet or lifestyle (e.g. hand-washing) might result in residual confounding.

In conclusion, we found that the overall adherence to the NNR was moderately good. Overall adherence to the
NNR was not associated with URTI risk in our study. However, when investigating individual components of the NNR, we found that high physical activity was associated with lower URTI risk. In addition, we found that the feasibility of using a web questionnaire at baseline and sending follow-up questionnaires via email was high in this population.

\section{Acknowledgements}

This study was financed in part by a grant from Osher Center for Integrative Medicine, Karolinska Institutet. Additional funds were received from the Swedish Council for Working Life and Social Research and the Swedish Research Council. The authors declare no conflict of interest. All authors contributed to the study design. K.B. supervised the study. K.B. and E.F obtained funding. K.B., O.B. and E.F. obtained the data. E.F. and S.C. performed data analysis and drafted the manuscript. All authors participated in critically revising the manuscript for important intellectual content. We thank Kenneth J. Rothman for methodological advice.

\section{References}

1. Doets EL, de Wit LS, Dhonukshe-Rutten RA et al. (2008) Current micronutrient recommendations in Europe: towards understanding their differences and similarities. Eur J Nutr 47, Suppl. 1, 17-40.

2. Nordiska ministerrådet (2004) Nordic Nutrition Recommendations 2004: Integrating Nutrition and Physical Activity, 4th ed. Copenhagen: Nordic Council of Ministers.

3. Fendrick AM, Monto AS, Nightengale B et al. (2003) The economic burden of non-influenza-related viral respiratory tract infection in the United States. Arch Intern Med 163, 487-494.

4. Grimsmo A, Hagman E, Faiko E et al. (2001) Patients, diagnoses and processes in general practice in the Nordic countries. An attempt to make data from computerised medical records available for comparable statistics. Scand J Prim Health Care 19, 76-82.

5. McCullough ML, Feskanich D, Rimm EB et al. (2000) Adherence to the Dietary Guidelines for Americans and risk of major chronic disease in men. Am J Clin Nutr $\mathbf{7 2}$, 1223-1231.

6. McCullough ML, Feskanich D, Stampfer MJ et al. (2000) Adherence to the Dietary Guidelines for Americans and risk of major chronic disease in women. Am J Clin Nutr $\mathbf{7 2}$, 1214-1222.

7. Kant AK, Schatzkin A, Graubard BI et al. (2000) A prospective study of diet quality and mortality in women. JAMA 283, 2109-2115.

8. Fung TT, Chiuve SE, McCullough ML et al. (2008) Adherence to a DASH-style diet and risk of coronary heart disease and stroke in women. Arch Intern Med 168, 713-720.

9. Imamura F, Jacques PF, Herrington DM et al. (2009) Adherence to 2005 Dietary Guidelines for Americans is associated with a reduced progression of coronary artery atherosclerosis in women with established coronary artery disease. Am J Clin Nutr 90, 193-201.

10. Shils ME \& Shike M (2006) Modern Nutrition in Health and Disease, 10th ed., pp. 670-684. Philadelphia, PA: Lippincott Williams \& Wilkins. 
11. Meydani SN, Han SN \& Hamer DH (2004) Vitamin E and respiratory infection in the elderly. Ann N Y Acad Sci 1031, 214-222.

12. Pauling L (1970) Vitamin C and the Common Cold. San Francisco, CA: W. H. Freeman.

13. Jackson JL, Lesho E \& Peterson C (2000) Zinc and the common cold: a meta-analysis revisited. J Nutr 130, $5 \mathrm{~S}$ Suppl., 1512S-1515S.

14. Prasad AS, Beck FW, Bao B et al. (2008) Duration and severity of symptoms and levels of plasma interleukin-1 receptor antagonist, soluble tumor necrosis factor receptor, and adhesion molecules in patients with common cold treated with zinc acetate. J Infect Dis 197, 795-802.

15. Balter KA, Balter O, Fondell E et al. (2005) Web-based and mailed questionnaires: a comparison of response rates and compliance. Epidemiology 16, 577-579.

16. Messerer M, Johansson SE \& Wolk A (2004) The validity of questionnaire-based micronutrient intake estimates is increased by including dietary supplement use in Swedish men. J Nutr 134, 1800-1805.

17. Michels KB, Holmberg L, Bergkvist L et al. (2001) Dietary antioxidant vitamins, retinol, and breast cancer incidence in a cohort of Swedish women. Int J Cancer 91, 563-567.

18. Lagerros YT, Mucci LA, Bellocco R et al. (2006) Validity and reliability of self-reported total energy expenditure using a novel instrument. Eur J Epidemiol 21, 227-236.

19. Linde A, Brytting M, Johansson M et al. (2004) The National Influenza Reference Centre, Swedish Institute for Infectious Disease Control, Annual Report September 2003-August 2004, p. 13. Solna: Swedish Institute for Infectious Disease Control.

20. Royston P (2007) Multivariable modeling with cubic regression splines: a principled approach. Stata J 7, 45-70.
21. Yngve A, Hambraeus L, Lissner L et al. (2006) The Women's Health Initiative. What is on trial: nutrition and chronic disease? Or misinterpreted science, media havoc and the sound of silence from peers? Public Health Nutr 9, 269-272.

22. Bingham SA, Luben R, Welch A et al. (2003) Are imprecise methods obscuring a relation between fat and breast cancer? Lancet 362, 212-214.

23. Becker W (1999) Dietary guidelines and patterns of food and nutrient intake in Sweden. Br J Nutr 81, Suppl. 2, S113-S117.

24. Bandini LG, Schoeller DA, Cyr HN et al. (1990) Validity of reported energy intake in obese and nonobese adolescents. Am J Clin Nutr 52, 421-425.

25. Prentice AM \& Jebb SA (1995) Obesity in Britain: gluttony or sloth? BMJ 311, 437-439.

26. Black AE, Coward WA, Cole TJ et al. (1996) Human energy expenditure in affluent societies: an analysis of 574 doublylabelled water measurements. Eur J Clin Nutr 50, 72-92.

27. Becker W \& Pearson M (2002) Riksmaten 1997-98. Befolkningens kostvanor och näringsintag. Metod-och resultatanalys (The Second National Food Consumption Survey 1997-98. Dietary Habits and Nutrient Intake in Sweden). Uppsala: National Food Administration.

28. Matthews CE, Ockene IS, Freedson PS et al. Moderate to vigorous physical activity and risk of upper-respiratory tract infection. Med Sci Sports Exerc 34, 1242-1248.

29. Mackinnon LT (2000) Chronic exercise training effects on immune function. Med Sci Sports Exerc 32, 7 Suppl., S369-S376.

30. Gwaltney JM Jr (2000) The common cold. In Principles and Practice of Infectious Diseases, 5th ed., pp. 651-656. [GL Mandell, RG Douglas, JE Bennett et al., editors]. Philadelphia, PA: Churchill Livingstone. 